\title{
Vertical Response of a Hereditary Deformable System
}

Botir Usmonov

Tashkent branch of Moscow State University, Tashkent, Uzbekistan

\begin{abstract}
An investigation of a viscoelastic material damping effect is studied on an example of plenum aircushion craft model. A numerical investigation was conducted to determine the vertical response characteristic of an open plenum air-cushion structure. The pure vertical motion of an air-cushion structure is investigated using a non-linear mathematical model; this incorporates a simple model to account hereditary deformable characteristic of the material.
\end{abstract}

Keywords - Viscoelasticity, hereditary deformable, aircushion, integro-differential equation.

\section{INTRODUCTION}

The term "Viscoelastic" material has quite a broad meaning. For example, in the literature [ 1 to 6] there is a use of this term if an equation of motion includes a viscous damping term; i.e., the equation of motion is written in terms of current instant

values of displacement, velocity, and acceleration. In this study, the equation of motion will include the integral term and history of strain is required for its formulation. Materials yielding such a constitutive relation (requiring history) are also called viscoelastic, but the term "hereditary" materials will be used in this study to distinguish them.

Hereditary properties are present in any composite material [1, 2 and 3]. The definition of the hereditary medium will give below. At this stage, we just note that the class of viscoelastic materials includes as a subclass the hereditary materials; i.e., these two terms are not identical.

In this study, numerical integration is applied for solution of the integro-differential equation. According to the correspondence (Volterra) principle, a solution of the viscoelastic problem can be obtained from a solution of the corresponding elastic problem be replacement of elasticity constants by their hereditary analogs (integral operators).

In this study, the use of exponentional terms for relaxation kernels is utilized.

\section{MODEL OF HEREDITARY DYNAMIC SYSTEM}

Consider disturbed motion of system near equilibrium with a view to sustain only vertical motion.

As to the constitutive relations, there are different models in use. Different models of viscoelastic material are discussed in references [4, 5, and 6].

In this study, we build model, which based on a constitutive law of the form:

$$
a \dot{\sigma}+\sigma=b \dot{y}+c y
$$

where

$\sigma=F \Delta P, \quad \theta=\frac{\rho_{0} V_{0}}{G_{0}}, \quad a=\frac{2 \theta P_{U_{0}}}{3 n P_{0}}, b=\frac{2 \theta P_{0}}{3 H}=\frac{2 m g \theta}{3 H}, \quad c=\frac{2 P_{U_{0}} F}{3 h_{0}}=\frac{2 m g}{3 h_{0}}$

In the references [1-6] are listed the different standard models of viscoelasticity. Therefore, viscoelastic system (1) have the properties of complex viscoelastic suspension and the relation between $\sigma$ and $y$, can be written as a hereditary type

of exponential kernel of relaxation

$$
\sigma=\frac{b}{a}\left[y(t)-\int_{0}^{t} R(t-\tau) y(\tau) d \tau\right],
$$

where

$$
R(t-\tau)=\left(\frac{1}{a}-\frac{c}{b}\right) e^{-\frac{1}{a}(t-\tau)}
$$

However the model of viscoelastic system (2) with exponential kernel

(3) includes creep strain, and stress relaxation, but have one major weakness such as $\dot{y}(t)$ in initial time has the final values, and do not fulfilled with experiment. This disadvantage easily overcome by use of weak-singularity features of the relaxation kernel (3) following from [3]:

$$
R(t-\tau)=\left(\frac{1}{a}-\frac{c}{b}\right) e^{-\frac{1}{a}(t-\tau)}(t-\tau)^{a-1}, \quad 0<a<1
$$


The fact, when model of the system is made of composite materials [4], then relation between $\sigma$ and $y$ must obey the law of hereditary non-linear theory viscoelasticity such:

$$
\sigma=\frac{b}{a}\left[\left(y(t)-\gamma y^{3}(t)\right)-\int_{0}^{t} R(t-\tau)\left[y(\tau)-\gamma y^{3}(t)\right] d \tau\right]
$$

The relation (5) is fairly common because in particularity can be obtained by standard model of viscoelastic body with kernels of relaxation (3). If considered that nonlinearity is $\gamma=0$, will be obtained known linear relation of the hereditary theory .

The equation of motion will be:

$$
m \ddot{y}(t)+\sigma=0
$$

Substituting (5) into (6) is built weak singular integrodifferential equation of nonlinear hereditary deformable system. This equation in dimensionless coordinates can be written in next form

$$
\ddot{U}(t)+\left[U(t)-\gamma U^{3}(t)\right]-(\mu-N) \int_{0}^{t} \Gamma(t-\tau)\left[U(\tau)-\gamma U^{3}(\tau)\right] d \tau=0
$$

where

$\mu=\frac{3 n P_{0}}{2 P_{U_{0}}}, \quad N=\frac{H}{h_{0}}, \quad \Gamma(t-\tau)=A_{0} e^{-\beta(t-\tau)}(t-\tau)^{a-1}$.

The initial conditions are

$$
U(0)=U_{0}, \quad \dot{U}(0)=\dot{U}_{0}
$$

The equation (7) with initial conditions (8) represent a mathematical model of hereditary deformable system.

\section{NUMERICAL EXAMPLE}

The results of calculations of steady state responses according to the expression of previous sections are presented below.

As example will be calculated vertical dynamic response of plenum air cushion craft. Both theoretical and experimental research $[9,10]$ has been performed to study the vertical motion and /or stability of various air cushion design configurations. The theoretical works [10] have been based on linear approaches to analyses air cushion vertical responses. Since air cushions are nonlinear, the application of linear analysis may be insufficient to predict fully the air cushion dynamic response behavior. Furthermore, any nonlinearity in air cushion response will have a direct bearing.

In this section presents the results of a numerical investigation to determine the dynamic behavior (in vertical) of a simple plenum air cushion suspension system in response to steady-state disturbances. The linear and nonlinear viscoelastic behavior of the system is examined.

An air-cushion craft structure is loaded by a vertical pressure load $P$, which is graphically presented in Figure 1 for a mass flow rate $Q\left(Q_{\text {in }}\right.$ and $\left.Q_{\text {out }}\right)$ to cushion.

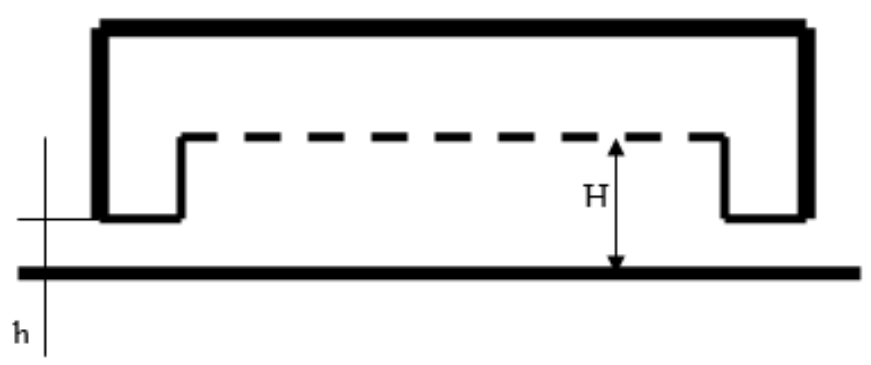

Fig.1: A Plenum Air Cushion scheme.

From this Figure 1 geometry of the air cushion represented by $h$-distance between ground and lower surface of the air cushion body and $H$ is average height of the air cushion. Motion parameters of the air cushion can be described by

$$
V=V_{0}\left(1-\frac{y}{H}\right) \text { and } h=h_{0}\left(1-\frac{y}{h_{0}}\right) \text {, }
$$

where $y=h_{0}-h$ (displacement of the air cushion).

Relation between inner volume pressure and density of air equal to $p \rho^{n}=$ const, where $n$ is polytrophic parameter of air.

By using equation (7) with initial conditions (8) is built mathematical model of the air cushion motion and stability problem. There are two unknowns: $U(t)$ and $N$. Therefore, it is needed to find the values of $N$ on which system is self-vibrating.

Exact solution of the equation (7) is not possible, so the numerical simulation of this equation with initial conditions (8) will be done by method, offered in [7]

$$
U_{n}=U_{0}+t_{n} U_{0}-\sum_{j=1}^{n-1} a_{j}\left(t_{n}-t_{j}\right)\left[U_{j}-\gamma U_{j}^{3}-\frac{(\mu-N) A_{0}}{a} \sum_{k=0}^{j} B_{k} e^{-\beta t_{n}}\left[U_{j-k}-\gamma U_{j-k}^{3}\right]\right],
$$


where

$$
\begin{aligned}
& t_{n}=n \Delta t, \quad a_{j}=\Delta t, \quad a_{0}=a_{n}=\Delta t / 2 \\
& j=1, n-1 ; \quad k=1, j-1 \\
& B_{0}=\Delta t^{a} / 2 ; \quad B_{j}=\Delta t^{a}\left[j^{a}-(j-1)^{a}\right] / 2 \\
& B_{k}=\Delta t^{a}\left[(k+1)^{a}-(k-1)^{a}\right] / 2
\end{aligned}
$$

To solve equation (10) is setup technical and kinematical characteristic of the air cushion as followed: $h_{0}$ - distance between lower surface of the air cushion and ground equal [0.02-0.12] meter; average height of the air cushion height is 1.25 meter; $n=1.4$ and pressure ratio is 1.2 . Rheological and nonlinearity parameters are varied for calculation of elastic, viscoelastic, and linear visa versa nonlinear cases.

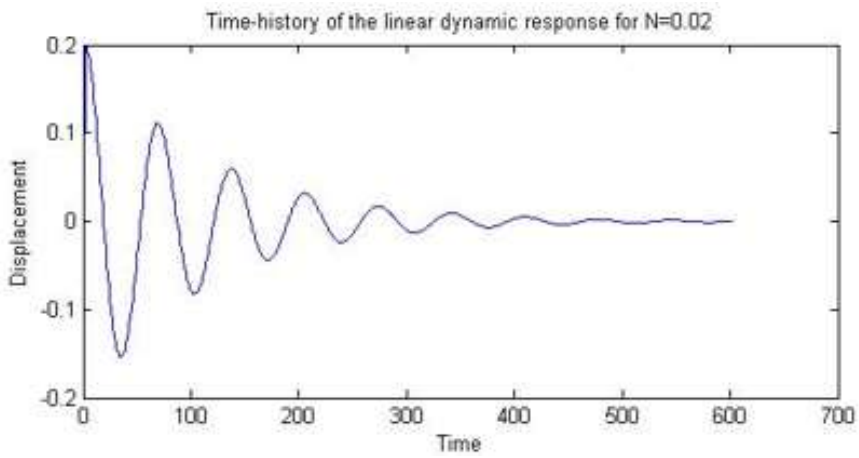

(a) linear case

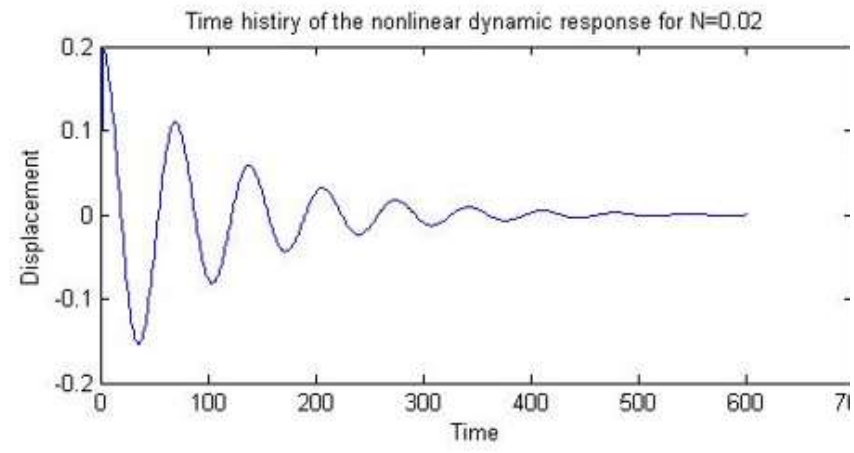

(b) nonlinear case

Fig.2: Time history of the vertical dynamics responses for

$$
N=0.02
$$

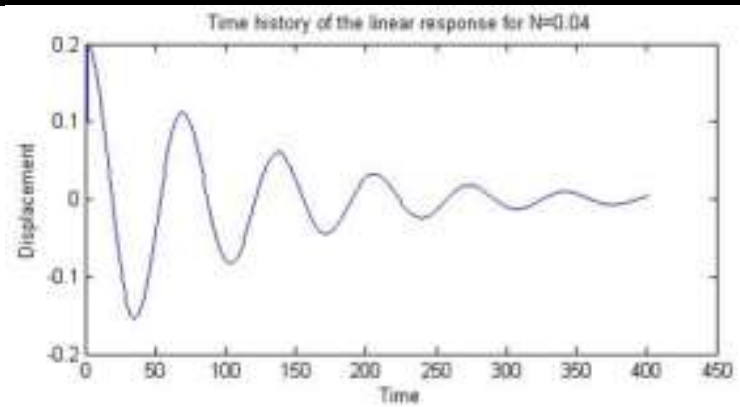

(a) linear case

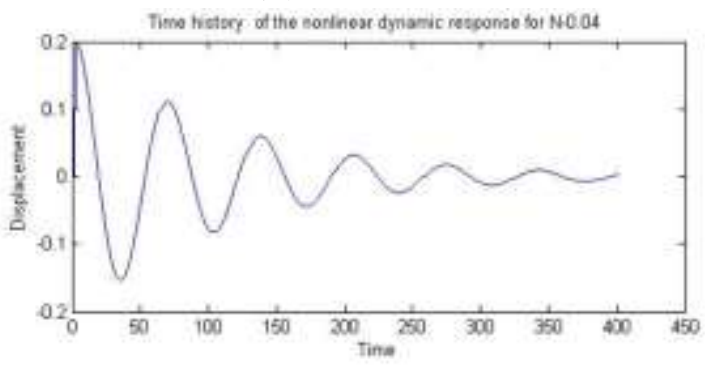

(b) nonlinear case

Fig.3: Time history of the vertical dynamics responses for

$$
N=0.04
$$

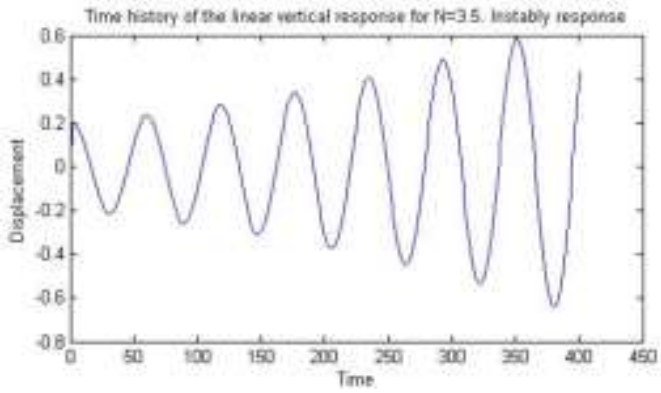

(a) linear case

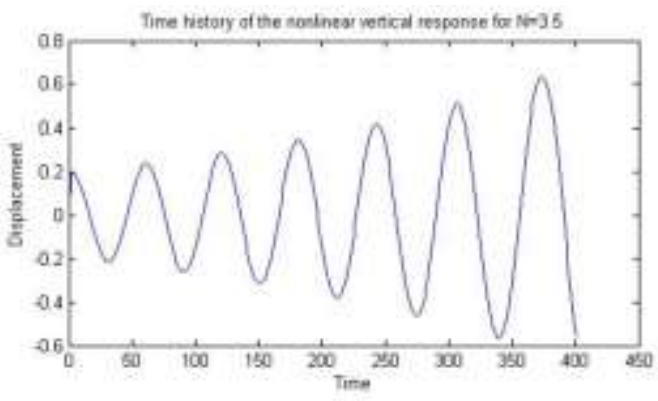

(b) nonlinear case

Fig.4: Time history of the vertical dynamics responses for

$$
N=3.5
$$




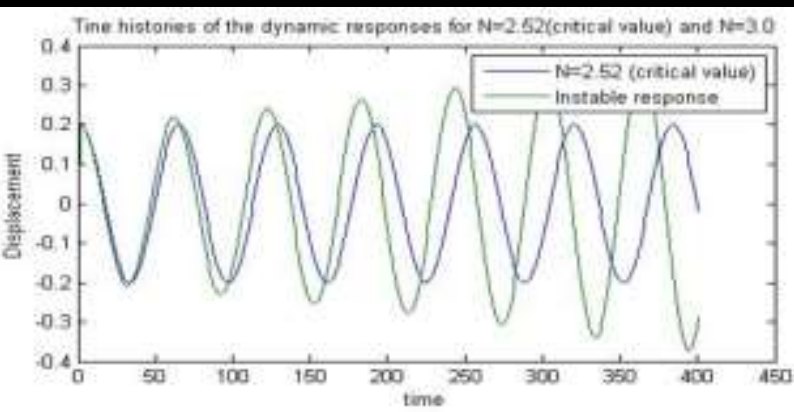

Fig.5: Comparison of dynamic responses.

From analysis of solutions of the equation (7) follows that $\mu-N>0$ in both linear and non-linear case is taking place damped oscillatory process. The damping speed and dissipation characteristics of the system are significantly dependent on the rheological parameters $A_{0}, \beta$ and $a$. When system has smaller the singularity parameter $a$ of the structure material, then damping properties of this material is higher. The self-vibrating is occurs only if $\mu-N<0$. Effect of physical non-linearity's and rheological parameters on the critical speed $N_{c r}$ is not difficult to show, through computer experiment based upon an algorithm (9).

\section{CONCLUSIONS}

Application of the numerical integration method to hereditary deformable problem is demonstrated. In this study the numerical solution in the time domain for dynamic problem (stability and flutter problem) have been presented.

The constitutive relation (stress-strain) was used in form of a hereditary law with the relation kernel represented by Abelian type function.

Numerical experiments for a problem of system bending vibration under force have been conducted. Finally, a understanding of viscoelastic phenomena may be exploited to damping in a manner that improves stability of the craft.

\section{REFERENCES}

[1] A.R. Rzanitsyn Creep theory. Moscow, Stroyizdat, 1986, pp 418

[2] Yu. N. Rabotnov 1980 Elements of Hereditary Solid Mechanics. Moscow: Mir.

[3] M. A. Koltunov Creep and relaxation Moscow Visshaya shkola, 1976, pp 276

[4] Badalov F B, Ganikhanov Sh F. Vibration of the hereditary deformable flight structure elements. Uzbekistan: TSAI; 2002
[5] V.D. Protasov, V.L. Starukhov, A.A. Kulkov Problems of application composite materials aerospace technique structures. Mechanics of composite materials \#6 1990, pp 1057-1063

[6] B. Gross 1953. Mathematical Structure of the theories of viscoelasticity. Paris: Hermann.

[7] R.M. Christensen 1982 Theory of Viscoelasticity. New York: Academic Press.

[8] F.B. Badalov Methods of solution integral and integrodifferential system, Tashkent, 1987, 296.

[9] C. W. Bert 1973 Journal of Sound and Vibration 29, 129-153. Material damping: an introductory review of mathematical models, measures and experimental techniques.

[10] Tulin, Marshall P. On the Vertical Motions of edge Jet Vehicles. Symp. On Ground effect phenomena, Dep. Aeronaut. Eng., Princeton Univ., Oct. 1959, pp.119134.

[11] Jack D. Leatherwood, Grayson V. Dixon, and David G. Stephens. Heave response of a plenum air cushion including passive and active control concepts, NASA technical Note, Langley Research Center Langley Station Hampton, Va., 1969

[12]Chung J. Skirt-material damping effects on Heave Dynamics of an air-cushion vehicle bag-and-finger skirt. Can. Aeronaut. Space J. Vol. 48, No.3, September 2002 